PROCEEDINGS OF THE

AMERICAN MATHEMATICAL SOCIETY

Volume 129, Number 3, Pages 699-711

S 0002-9939(00)05976-1

Article electronically published on November 3, 2000

\title{
A CAPACITARY WEAK TYPE INEQUALITY FOR SOBOLEV FUNCTIONS AND ITS APPLICATIONS
}

\author{
WEI-SHYAN TAI
}

(Communicated by Palle E. T. Jorgensen)

\begin{abstract}
In this paper a capacitary weak type inequality for Sobolev functions is established and is applied to reprove some well-known results concerning Lebesgue points, Taylor expansions in the $L^{p}$-sense, and the Lusin type approximation of Sobolev functions.
\end{abstract}

\section{INTRODUCTION}

In [15] Ziemer obtained a capacitary weak type inequality for maximal functions of Sobolev functions as follows.

Theorem 1.1. If $1<p<\infty, R<1$, and $k$ is a positive integer such that $k p<n$, then there is a positive constant $C$ depending on $n, k, p$ and $R$ such that

$$
B_{k, p}\left(\left\{x \in \mathbf{R}^{n}: M_{p, R} u(x)>\lambda\right\}\right) \leq C\|u\|_{k, p}^{p} / \lambda^{p}
$$

for $u \in W^{k, p}\left(\mathbf{R}^{n}\right)$ and $\lambda>0$, where $B_{k, p}$ is the Bessel capacity and

$$
M_{p, R} u(x)=\sup _{0<r<R}\left(f_{B(x, r)}|u(y)|^{p} d y\right)^{1 / p} .
$$

The proof uses the representation of Sobolev functions as Bessel potentials (see Calderón [4] or Stein [13]) and the Hardy-Littlewood maximal theorem (see Stein [13]).

Our purpose in this paper is to establish the following analogous inequality:

Theorem 1.2. Let $k$ be a positive integer with $k p<n$ and let $p^{*}=n p /(n-k p)$, where $1 \leq p<\infty$. Then, there exists a positive constant $C$ depending on $n, k$ and $p$ such that

$$
R_{k, p}\left(\left\{x \in \mathbf{R}^{n}: M_{p^{*}} u(x)>\lambda\right\}\right) \leq C\|u\|_{k, p}^{p} / \lambda^{p}
$$

for $u \in W^{k, p}\left(\mathbf{R}^{n}\right)$ and $\lambda>0$, where $R_{k, p}$ is the Riesz capacity and

$$
M_{p^{*}} u(x)=\sup _{r>0}\left(f_{B(x, r)}|u(y)|^{p^{*}} d y\right)^{1 / p^{*}} .
$$

Received by the editors May 28, 1996.

2000 Mathematics Subject Classification. Primary 26B35, 41A30, 46E35.

Key words and phrases. Sobolev functions, Riesz capacities, Lusin type properties.

This work was partially supported by Academia Sinica-Taipei, Taiwan, R.O.C. The author is deceased. 
Our proof is based on an elementary integral formula for functions in $W^{1, p}\left(\mathbf{R}^{n}\right)$ (see Lemma 3.1) and Poincaré's inequality. Notice that the maximal function here is raised to a larger power than that in Theorem 1.1 and that the result holds also for $p=1$. It is worthwhile to observe here that although Riesz and Bessel capacities have the same null sets, they are not equal; actually for balls of large radius we have

$$
B_{\alpha, p} \sim\left[R_{\alpha, p}\right]^{n /(n-\alpha p)} .
$$

For this see the remark after Theorem 1 in 2 . Theorem 1.2 will be proved in Section 3 while necessary preliminaries are collected in Section[2.

As in Ziemer [15], we apply this inequality to reprove some well-known results with some modifications concerning Lebesgue points and Taylor expansions in the $L^{p}$-sense for a Sobolev function (see Theorem 4.1 and Theorem 4.3 ).

Furthermore, we can also provide a simpler and direct method to establish the Lusin type approximation in Riesz capacity for Sobolev functions in Section 4 The idea goes back to Liu 8. According to Theorem 4.3, a Sobolev function is uniformly differentiable in the $L^{p}$-sense on the complement of sets with small Riesz capacity and Lebesgue measure, and the remainder terms of its Taylor expansions in the $L^{p}$-sense can be dominated by the sum of maximal functions for its weak derivatives. If we apply Theorem 1.2 and the Hardy-Littlewood maximal theorem to control these maximal functions of weak derivatives carefully, we can find the approximating functions by using a lemma due to Calderón and Zygmund (Lemma 4.5) and a variant of the Whitney extension theorem endowed with norms (Theorem 2.1). Thus, we give an alternative proof of the Michael-Ziemer theorem for Sobolev functions (Michael and Ziermer 12]) (see Theorem 4.4).

\section{Preliminaries}

Let $\Omega$ be an open subset of $\mathbf{R}^{n}$. By $W^{k, p}(\Omega)$ we denote the usual Sobolev space with the norm

$$
\|u\|_{k, p ; \Omega}=\sum_{|\alpha| \leq k}\left\|D^{\alpha} u\right\|_{p ; \Omega},
$$

for its elements $u$, where $1 \leq p \leq \infty$ and $k$ is a nonnegative integer.

We use $|\cdot|$ to denote either Euclidean norm in various dimensions or Lebesgue (outer) measures of sets in $\mathbf{R}^{n}$; its connotation will be clear from the context. We write

$$
f_{B(x, r)} u(y) d y=\frac{1}{|B(x, r)|} \int_{B(x, r)} u(y) d y,
$$

where $B(x, r)$ is the open ball with center $x$ and radius $r$.

For a locally integrable function $f$ on $\mathbf{R}^{n}$, the function

$$
(M f)(x)=\sup _{r>0} f_{B(x, r)}|f(Y)| d y
$$

is called the Hardy-Littlewood maximal function of $f$. It is a well-known result that

$$
\left|\left\{x \in \mathbf{R}^{n}:(M f)(x)>\lambda\right\}\right| \leq C(n)\|f\|_{p}^{p} / \lambda^{p}
$$


and

$$
\lim _{\lambda \rightarrow \infty} \lambda^{p}\left|\left\{x \in \mathbf{R}^{n}:(M f)(x)>\lambda\right\}\right|=0
$$

for $f \in L^{p}\left(\mathbf{R}^{n}\right)$ and $\lambda>0$, where $1 \leq p<\infty$. See Stein [13] for proofs. We also adopt the notation

$$
\left(M_{p} f\right)(x)=\sup _{r>0}\left(f_{B(x, r)}|f(y)|^{p} d y\right)^{1 / p}
$$

for $f \in L^{p}\left(\mathbf{R}^{n}\right), 1 \leq p<\infty$. Obviously, $m_{1} f=M f$.

We recall that the Riesz potential $I_{\alpha} f$ of $f$ is defined by

$$
\left(I_{\alpha} f\right)(x)=\gamma(\alpha)^{-1} \int_{\mathbf{R}^{n}}|x-y|^{-n+\alpha} f(y) d y
$$

with $\gamma(\alpha)=\pi^{n / 2} \Gamma(\alpha / 2) / \Gamma\left(\frac{n}{2}-\frac{\alpha}{2}\right), 0<\alpha<n$. For $\alpha>0, p \geq 1$ with $\alpha p<n$, the Riesz capacity $R_{\alpha, p}$ is defined by

$$
R_{\alpha, p}(E)=\inf \left\{\|f\|_{p}^{p}:\left(I_{\alpha} f\right)(x) \geq 1 \text { on } E, f \geq 0\right\}
$$

for $E \subseteq \mathbf{R}^{n}$. In the following we list some basic properties of Riesz capacity:

(i) $R_{\alpha, p}$ is an outer measure.

(ii) $R_{\alpha, p}(B(x, r))=R_{\alpha, p}(B(0 ; 1)) r^{n-\alpha p}$ for $x \in \mathbf{R}^{n}$ and $r>0$.

(iii) $R_{\alpha, p}(E)=\inf \left\{R_{\alpha, p}(U): E \subseteq U, U\right.$ open $\}$ for $E \subseteq \mathbf{R}^{n}$.

(iv) $|E|^{p / p^{*}} \leq C(n, p, \alpha) R_{\alpha, p}(E)$ for $E \subseteq \mathbf{R}^{n}$, where $p^{*}=n p /(n-\alpha p)$.

Moreover, we also have $R_{\alpha, p}(E)=0$ implies $H^{n-\alpha p+\varepsilon}(E)=0$ for $\varepsilon>0$, where $H^{s}$ denotes the $s$-dimensional Hausdorff measure on $\mathbf{R}^{n}$. We refer to Ziemer [16] for further information. (We do not know whether $R_{\alpha, p}$ is a Choquet capacity when $p=1$; but all the above properties hold in this case.)

Finally, we state the Whitney extension theorem. Let $F \subseteq \mathbf{R}^{n}$. A function $u$ defined on $F$ belongs to $t^{k}(F), k$ a nonnegative integer, if there exist a nonnegative number $M$ and a family of polynomials $\left\{P_{x}\right\}_{x \in F}$ of degree at most $k$ so that

1) $u(x)=P_{x}(x)$ for $x \in F$,

2) $\left|D^{\alpha} P_{x}(x)\right| \leq M$ and $\left|D^{\alpha} P_{y}(y)-D^{\alpha} P_{x}(y)\right| \leq M|x-y|^{k-|\alpha|}$ for $|\alpha| \leq k, x, y \in$ $F$,

3) $\lim _{y \rightarrow x}\left|D^{\alpha} P_{y}(y)-D^{\alpha} P_{y}(x)\right| /|y-x|^{k-|\alpha|}=0$ uniformly on each compact subset of $F$.

The smallest such $M$ is denoted by $\|u\|_{t^{k}(F)}$. Since our version of the Whitney extension theorem is slightly different from that in Ziemer [16], we shall sketch the proof for the convenience of readers.

Theorem 2.1 (Whitney 14]). Let $F$ be a closed subset of $\mathbf{R}^{n}$ and let $k$ be a nonnegative integer. Suppose $u \in t^{k}(F)$. Then, for each $\varepsilon>0$, there exists $\bar{u} \in C^{k}\left(\mathbf{R}^{n}\right)$ such that $\left.\bar{u}\right|_{F}=u$ in $t^{k}(F)$ (i.e. $D^{\alpha} P_{x}(x)=D^{\alpha} \bar{u}(x)$ for $\left.x \in F,|\alpha| \leq k\right)$; moreover,

$$
\|\bar{u}\|_{t^{k}\left(\mathbf{R}^{n}\right)} \leq C\|u\|_{t^{k}(F)}
$$

and

$$
\operatorname{supp} \bar{u} \subseteq\left\{x \in \mathbf{R}^{n}: \operatorname{dist}(x, F)<\varepsilon\right\},
$$

where $C$ is a positive constant depending only on $n, k$ and $\varepsilon$. (In what follows we always take $\varepsilon=1$.) 
Sketch of the proof. Following the proof of Federer [6] 3.1.14], we can construct a function $\tilde{u} \in C^{k}\left(\mathbf{R}^{n}\right)$ such that

$$
\left|D^{\alpha} \tilde{u}(x)-D^{\alpha} P_{b}(x)\right| \leq C(n, k)\|u\|_{t^{k}(F)}|x-b|^{k}
$$

for $x \in \mathbf{R}^{n}$ with $\operatorname{dist}(x, F)<1 / 3$, where $b \in F$ with $|x-b|=\operatorname{dist}(x, F)$. Therefore,

$$
\left|D^{\alpha} u(x)\right| \leq C(n, k)\|u\|_{t^{k}(F)}
$$

for $x \in \mathbf{R}^{n}$ with $\operatorname{dist}(x, F)<1 / 3$.

Note that for any given $\varepsilon>0$ we can find a function $\varphi_{\varepsilon} \in C^{\infty}\left(\mathbf{R}^{n}\right)$ satisfying

(i) $\operatorname{supp} \varphi_{\varepsilon} \subseteq\left\{x \in \mathbf{R}^{n}: \operatorname{dist}(x, F)<\varepsilon\right\}$;

(ii) $0 \leq \varphi_{\varepsilon} \leq 1, \varphi_{\varepsilon}=1$ on $\left\{x \in \mathbf{R}^{n}\right.$ : $\left.\operatorname{dist}(x, F)<\varepsilon / 2\right\}$; and

(iii) $\left|D^{\alpha} \varphi_{\varepsilon}(x)\right| \leq C(n,|\alpha|) \varepsilon^{-|\alpha|}$ for $x \in \mathbf{R}^{n}$.

Now, without loss of generality, we may assume $\varepsilon<1 / 3$. Let $\bar{u}=\varphi_{\varepsilon} \tilde{u}$. Then, $\bar{u} \in C^{k}\left(\mathbf{R}^{n}\right), \operatorname{supp} \bar{u} \subseteq\left\{x \in \mathbf{R}^{n}: \operatorname{dist}(x, F)<\varepsilon\right\}$ and $\|\bar{u}\|_{k, \infty} \leq C(n, k, \varepsilon)\|u\|_{t^{k}(F)}$ for $|\alpha| \leq k$. Hence, $\bar{u}$ is just as desired.

When $F$ is a compact set, we refer to Malgrange [10] for a detailed proof.

\section{Proof of Theorem 1.2}

For the proof of Theorem 1.2 we need the following lemmas:

Lemma 3.1. For $u \in W^{1, p}\left(\mathbf{R}^{n}\right), 1 \leq p<n$,

$$
u(y)=\frac{1}{\omega_{n}} \int_{\mathbf{R}^{n}} \frac{\langle y-z, D u(z)\rangle}{|y-z|^{n}} d z
$$

holds for almost every $y \in \mathbf{R}^{n}$, where $\omega_{n}$ is the area of the unit sphere in $\mathbf{R}^{n}$. (In particular, (3.1) holds whenever $y$ is a Lebesgue point of $u$ and $M(|D u|)(x)<\infty$.)

We refer to Stein [13] for the proof.

Lemma 3.2. Let $u \in W^{1, p}\left(\mathbf{R}^{n}\right), 1 \leq p<n$. Then

$$
I_{\alpha}(|u|)(x) \leq C(n, \alpha) I_{\alpha+1}(|D u|)(x)
$$

for all $x \in \mathbf{R}^{n}$, where $0<\alpha<n-1$.

Proof. First, we note that

$$
\int_{\mathbf{R}^{n}}|x-y|^{-n+\alpha}|y|^{-n+\beta} d y=C(n, \alpha, \beta)|x|^{-n+\alpha+\beta} \quad(x \neq 0)
$$

if $\alpha, \beta>0, \alpha+\beta<n$, since

$$
x \in \mathbf{R}^{n} \backslash\{0\} \mapsto \int_{F^{n}}|x-y|^{-n+\alpha}|y|^{-n+\beta} d y
$$

is rotation-invariant and homogeneous of degree $-n+\alpha+\beta$. Besides, from Lemma 3.1, we have

$$
|u(x)| \leq C(n) I_{1}(|D u|)(x)
$$


for almost every $x \in \mathbf{R}^{n}$ if $u \in W^{1, p}\left(\mathbf{R}^{n}\right), 1 \leq p<n$. Applying (3.2) (with $\beta=1$ ) to (3.3), by Fubini's theorem,

$$
\begin{aligned}
\int_{\mathbf{R}^{n}}|x-y|^{-n+\alpha}|u(y)| d y \\
\leq C(n) \int_{\mathbf{R}^{n}}|x-y|^{-n+\alpha}\left(\int_{\mathbf{R}^{n}}|y-z|^{-n+1}|D u(z)| d z\right) d y \\
=C(n) \int_{\mathbf{R}^{n}}\left(\int_{\mathbf{R}^{n}}|z-y|^{-n+\alpha}|y-z|^{-n+1} d y\right)|D u(z)| d z \\
\quad=C(n) \int_{\mathbf{R}^{n}}\left(\int_{\mathbf{R}^{n}}|(x-z)-y|^{-n+\alpha}|y|^{-n+1} d y\right)|D u(z)| d z \\
\leq C(n, \alpha) \int_{\mathbf{R}^{n}}|x-z|^{-n+\alpha+1}|D u(z)| d z .
\end{aligned}
$$

The proof is complete.

Lemma 3.3. Let $k$ and $n$ be positive integers with $k p<n$ and let $p^{*}=n p /(n-k p)$, where $1 \leq p<\infty$. Suppose $u \in W^{k, p}\left(\mathbf{R}^{n}\right)$. Then,

$$
\left(M_{p^{*}} u\right)(x) \leq C \sum_{|\alpha|=k}\left\{\sup _{r>0} r^{k}\left(f_{B(x, r)}\left|D^{\alpha} u(y)\right|^{p} d y\right)^{1 / p}+I_{k}\left(\left|D^{\alpha} u\right|\right)(x)\right\}
$$

for all $x \in \mathbf{R}^{n}$, where $C$ is a positive constant depending on $n, k$ and $p$.

Proof. First, we claim that

$$
\begin{aligned}
\sup _{\rho>0} \rho^{k} & \left(f_{B(x, \rho)}|u(y)|^{p} d y\right)^{1 / p} \\
\leq & C(n, k, p)\left\{\sup _{\rho>0} \rho^{k+1}\left(f_{B(x, \rho)}|D u(y)|^{p} d y\right)^{1 / p}+I_{k+1}(|D u|)(x)\right\}
\end{aligned}
$$

for $u \in W^{1, p}\left(\mathbf{R}^{n}\right)$ and $x \in \mathbf{R}^{n},(k+1) p<n, 1 \leq p<\infty, k$ a nonnegative integer. For $\rho>0$, we set

$$
u_{1}(y)=\frac{1}{\omega_{n}} \int_{B(x, 2 \rho)} \frac{\langle y-z, D u(z)\rangle}{|y-z|^{n}} d z
$$

and

$$
u_{2}(y)=\frac{1}{\omega_{n}} \int_{\mathbf{R}^{n} \backslash B(x, 2 \rho)} \frac{\langle y-z, D u(z)\rangle}{|y-z|^{n}} d z .
$$


Then, from Lemma 3.2, $u(y)=u_{1}(y)+u_{2}(y)$ holds for almost all $y \in \mathbf{R}^{n}$. By Minkowski's inequality,

$$
\begin{aligned}
& \left(\int_{B(x, \rho)}\left|u_{1}(y)\right|^{p} d y\right)^{1 / p} \\
& \quad \leq \omega_{n}^{-1}\left(\int_{B(x, \rho)}\left(\int_{B(x, 2 \rho)}|y-z|^{-n+1}|D u(z)| d z\right)^{p} d y\right)^{1 / p} \\
& \quad \leq \omega_{n}^{-1}\left(\int_{B(x, \rho)}\left(\int_{B(x, 3 \rho)}|D u(y+z)||z|^{-n+1} d z\right)^{p} d y\right)^{1 / p} \\
& \quad \leq \omega_{n}^{-1} \int_{B(x, 3 \rho)}\left(\int_{B(x, \rho)}|D u(y+z)|^{p} d z\right)|z|^{-n+1} d z \\
& \quad \leq \omega_{n}^{-1} \int_{B(x, 3 \rho)}\left(\int_{B(x, 4 \rho)}|D u(y)|^{p} d y\right)^{1 / p}|z|^{-n+1} d z \\
& \quad \leq 3 n \rho\left(\int_{B(x, 4 \rho)}|D u(y)|^{p} d y\right)^{1 / p} .
\end{aligned}
$$

Now if $y \in B(x, \rho)$ and $z \in \mathbf{R}^{n} \backslash B(x, 2 \rho)$, we have

$$
|y-z| \geq|z-x|-|y-x| \geq|z-x|-\rho \geq|z-x| / 2 \geq \rho .
$$

It follows that

$$
\begin{aligned}
\left|u_{2}(y)\right| & \leq \omega_{n}^{-1} \int_{|x-z| \geq 2 \rho}|y-z|^{-n+1}|D u(z)| d z \\
& \leq 2^{n-1} \omega_{n}^{-1} \int_{|x-z| \geq 2 \rho}|x-z|^{-n+1}|D u(z)| d z
\end{aligned}
$$

for $y \in B(x, \rho)$. Hence,

$$
\begin{aligned}
\rho^{k}\left(f_{B(x, \rho)}\left|u_{2}(y)\right|^{p} d y\right)^{1 / p} & \leq 2^{n-1} \rho^{k} \omega_{n}^{-1} \int_{|x-z| \geq 2 \rho}|x-z|^{-n+1}|D u(z)| d z \\
& \leq C(n, k) I_{k+1}(|D u|)(x) .
\end{aligned}
$$

Combining (3.6) and (3.7), we have established the inequality (3.5).

Next, we claim that

$$
\begin{aligned}
& \sup _{r>0} r^{k}\left(f_{B(x, r)}|u(y)|^{p^{*}} d y\right)^{1 / p^{*}} \\
& \leq C(n, p, k)\left\{\sup _{r>0} r^{k+1}\left(f_{B(x, r)}|D u(y)|^{p} d y\right)^{1 / p}+I_{k+1}(|D u|)(x)\right\}
\end{aligned}
$$


for $u \in W^{1, p}\left(\mathbf{R}^{n}\right), 1 \leq p<n, k \geq 0,(k+1) p<n$, and $p^{*}=n p /(n-k p)$. By Poincaré's inequality and Hölder's inequality, for $\rho>0$,

$$
\begin{gathered}
\left(f_{B(x, \rho)}|u(y)|^{p^{*}} d y\right)^{1 / p^{*}} \leq C(n, p) \rho\left(f_{B(x, \rho)}|D u(y)|^{p} d y\right)^{1 / p}+f_{B(x, \rho)}|u(y)| d y \\
\leq C(n, p) \rho\left(f_{B(x, \rho)}|D u(y)|^{p} d y\right)^{1 / p}+\left(f_{B(x, \rho)}|u(y)|^{p} d y\right)^{1 / p} .
\end{gathered}
$$

Therefore, from (3.5) and (3.9), (3.8) is established.

Now, it is easy to verify that (3.4) follows from (3.8) and Lemma 3.2 just by induction.

Lemma 3.4. Let $\alpha>0$ with $\alpha p<n$, where $1 \leq p<\infty$. Then, there exists a positive constant $C$ depending only on $n, p$ and $\alpha$ such that

$$
R_{\alpha, p}\left(\left\{x \in \mathbf{R}^{n}: \sup _{r>0} r^{\alpha}\left(f_{B(x, r)}|f(y)|^{p} d y\right)^{1 / p}>\lambda\right\}\right) \leq C\|f\|_{p}^{p} / \lambda^{p}
$$

for $f \in L^{p}\left(\mathbf{R}^{n}\right)$ and $\lambda>0$.

Proof. For $\lambda>0$, let $E_{\lambda}=\left\{x \in \mathbf{R}^{n}: \sup _{r>0} r^{\alpha}\left(f_{B(x, r)}|f(y)|^{p} d y\right)^{1 / p}>\lambda\right\}$. Then, for each $x \in E_{\lambda}$, there exists a positive number $r=r(x)$ such that

$$
\int_{B(x, r)}|f(y)|^{p} d y \geq \lambda^{p}|B(0 ; 1)| r^{n-\alpha p} .
$$

Let $\mathcal{F}$ be the collection of all balls which satisfy (3.10). Then

$$
\sup _{B \in \mathcal{F}} \operatorname{diam} B \leq 2\left(\|f\|_{p}^{p} / \lambda^{p}|B(0 ; 1)|\right)^{1 /(n-\alpha p)}<\infty
$$

and $E_{\lambda} \subseteq \bigcup_{B \in \mathcal{F}} B$. Thus, by Vitali's covering lemma (see Stein [13, Chapter 1]), there exists a countable disjoint subfamily $S$ of $\mathcal{F}$ such that

$$
E_{\alpha} \subseteq \bigcup_{B \in \rho} \widehat{B}
$$

where $\widehat{B}$ denotes the ball with radius five times that of $B$ but with the same center. Consequently,

$$
\begin{aligned}
R_{\alpha, p}\left(E_{\lambda}\right) \leq \sum_{B \in S} R_{\alpha, p}(\widehat{B}) & =C \sum_{B(x, r) \in S}(5 r)^{n-\alpha p} \\
& \leq \frac{C}{\lambda^{p}} \sum_{B \in S} \int_{B}|f(y)|^{p} d y \quad \text { (by (3.10)) } \\
& =\frac{C}{\lambda^{p}} \int_{\bigcup_{B \in S} B}|f(y)|^{p} d y \\
& \leq C\|f\|_{p}^{p} / \lambda^{p} .
\end{aligned}
$$

We have established this lemma. 
We are now in a position to prove Theorem 1.2. For $u \in W^{k, p}\left(\mathbf{R}^{n}\right), k p<n$, by Lemma 3.3. (3.4) holds for all $x \in \mathbf{R}^{n}$. Thus, by the property (i) of Riesz capacity stated in Section 2, one gets that

$$
\begin{aligned}
R_{\alpha, p}\left(\left\{x \in \mathbf{R}^{n}:\left(M_{p^{*}} u\right)(x)>\lambda\right\}\right) \\
\leq R_{\alpha, p}\left(\left\{x \in \mathbf{R}^{n}: C \sum \sup _{r>0} r^{k}\left(f_{B(x, r)}\left|D^{\alpha} u(y)\right|^{p} d y\right)^{1 / p}>\lambda / 2\right\}\right) \\
\quad+R_{\alpha, p}\left(\left\{x \in \mathbf{R}^{n}: C \sum_{|\alpha|=k} I_{k}\left(\left|D^{\alpha} u\right|\right)(x)>\lambda / 2\right\}\right) \\
\equiv I+I I .
\end{aligned}
$$

By Lemma 3.4, $I \leq C(n, p, k)\|u\|_{k, p}^{p} / \lambda^{p}$, and, from the definition of Riesz capacity, $I I \leq C(n, p, k)\|u\|_{k, p}^{p} / \lambda^{p}$. Combining these estimates,

$$
R_{\alpha, p}\left(\left\{x \in \mathbf{R}^{n}:\left(M_{p^{*}} u\right)(x)>\lambda\right\}\right) \leq C(n, p, k)\|u\|_{k, p}^{p} / \lambda^{p} .
$$

This completes the proof.

Remark 3.5. Following the proof of the inequality (3.5), we can show that

$$
M_{p}\left(I_{\alpha} f\right)(x) \leq C(n, \alpha, p)\left\{\sup _{r>0} r^{\alpha}\left(f_{B(x, r)}|f(y)|^{p} d y\right)^{1 / p}+I_{\alpha}(|f|)(x)\right\}
$$

for $f \in L^{p}\left(\mathbf{R}^{n}\right)$ and $x \in \mathbf{R}^{n}$, where $0<\alpha, \alpha<n, 1 \leq p<\infty$. Consequently, following the proof of Theorem 1.2 , we have

$$
R_{\alpha, p}\left(\left\{x \in \mathbf{R}^{n}: M_{p}\left(I_{\alpha} f\right)(x)>\lambda\right\}\right) \leq C(n, \alpha, p)\|f\|_{p}^{p} / \lambda^{p}
$$

for all $\lambda>0$.

\section{Applications}

In this section, we employ Theorem 1.2 to reprove some well-known results concerning Lebesgue points and Taylor expansions in the $L^{p}$-sense for Sobolev functions. Besides, we also provide an alternative proof of the Michael-Ziemer theorem of Lusin type approximation for Sobolev functions.

We first consider Lebesgue points of a Sobolev function.

Theorem 4.1. Let $k$ be a positive integer such that $k p<n$, where $1 \leq p<\infty$. Then, for $u \in W^{k, p}\left(\mathbf{R}^{n}\right)$, the following statements hold:

(i) There exists a Borel set $E$ with $R_{k, p}(E)=0$ such that

$$
u^{*}(x)=\lim _{r \rightarrow 0} f_{B(x, r)} u(y) d y
$$

exists and

$$
\lim _{r \rightarrow 0} f_{B(x, r)}\left|u(y)-u^{*}(x)\right|^{p^{*}} d y=0
$$

for all $x \in \mathbf{R}^{n} \backslash E$ (cf. Federer and Ziemer [7], Babgy and Ziemer [3], and Michael and Ziemer [12]). 
(ii) For each $\varepsilon>0$ there exists a closed set $R$ such that $R_{k, p}\left(\mathbf{R}^{n} \backslash F\right)<\varepsilon$ and

$$
\lim _{r \rightarrow 0} f_{B(x, r)}\left|u(y)-u^{*}(x)\right|^{p^{*}} d y=0
$$

uniformly on F (cf. Ziemer [15]).

It is worthwhile to note here that owing to the remarks following Theorem 1.2 the statement (ii) is stronger than that in Ziemer [15]. Since this theorem can be proved by straightforward modifications of methods used in Stein [13, p. 8] and Ziemer [16, Theorem 1.3.8 and Theorem 3.10.2] together with Theorem 1.2, we omit the proof.

Remark 4.2. According to the property (iv) of Riesz capacity stated in Section 2 the statement (ii) can be rewritten in the following form: for each $\varepsilon>0$ there exists a closed set $R$ such that

$$
R_{k, p}\left(\mathbf{R}^{n} \backslash F\right)<\varepsilon \quad\left(\left|\mathbf{R}^{n} \backslash F\right|<\varepsilon\right)
$$

and

$$
\lim _{r \rightarrow 0} f_{B(x, r)}\left|u(y)-u^{*}(x)\right|^{p^{*}} d y=0
$$

uniformly on $F$.

In fact, this follows directly from Theorem 1.2 and (2.1).

Next, we study Taylor expansions in the $L^{p}$-sense of Sobolev functions. Let $k, m$ be integers such that $0 \leq m<k,(k-m) p<n$ and $p^{*}=n p /[n-(k-m) p]$, where $1 \leq p<\infty$. Then, from Theorem 4.1 for $u \in W^{k, p}\left(\mathbf{R}^{n}\right)$, there exists a set $E \subseteq \mathbf{R}^{n}$ such that

$$
R_{k-m ; p}(E)=0(=|E|)
$$

and

$$
\lim _{r \rightarrow 0} f_{B(x, r)}\left|D^{\alpha} u(y)-\left(D^{\alpha} u\right)^{*}(x)\right|^{p^{*}} d y=0
$$

for all $x \in \mathbf{R}^{n} \backslash E,|\alpha| \leq m$, where $\left(D^{\alpha} u\right)^{*}(x)$ is some real number. By the Lebesgue differentiation theorem, $\left(D^{\alpha} u\right)(x)=\left(D^{\alpha} u\right)^{*}(x)(|\alpha| \leq m)$ for almost all $x \in \mathbf{R}^{n}$. Therefore, by abuse of notation, in what follows we still denote $\left(D^{\alpha} u\right)^{*}(x)$ by $D^{\alpha} u(x)$. Set

$$
P_{x}(y)=\sum_{|\alpha| \leq m} \frac{D^{\alpha} u(x)}{\alpha !}(y-x)^{\alpha} .
$$

Then the Taylor polynomial $P_{z}$ is well defined for all $x \in \mathbf{R}^{n}$ except for a set $E$ with $R_{k-m, p}(E)=0$. As a consequence of Theorem 4.1 we have the following wellknown result with some modifications, the proof of which follows known techniques used in Ziemer [16, Theorem 3.4.1 and Theorem 3.4.2] together with Theorem 1.2

Theorem 4.3. Let $k, m$ be integers with $0 \leq m<k,(k-m) p<n$, where $1 \leq$ $p<\infty$, and let $p^{*}=n p /[n-(k-m) p]$. For $u \in W^{k, p}\left(\mathbf{R}^{n}\right)$, we set $P_{x}^{(m)}(y)=$ $\sum_{|\alpha| \leq m} \frac{D^{\alpha} u(x)}{\alpha !}(y-x)^{\alpha}$. Then, the following statements hold: 
(i) There exists a Borel set $E \subseteq \mathbf{R}^{n}$ with $R_{k-m, p}(E)=0$ such that

$$
\sup _{r>0} r^{-m}\left(f_{B(x, r)}\left|u(y)-P_{x}^{(m)}(y)\right|^{p^{*}} d y\right)^{1 / p^{*}} \leq 2 \sum_{|\alpha|-m} M_{p^{*}}\left(\left|D^{\alpha} u\right|\right)(x)
$$

and

$$
\lim _{r \rightarrow 0} r^{-m}\left(f_{B(x, r)}\left|u(y)-P_{x}^{(m)}(y)\right|^{p^{*}} d y\right)^{1 / p^{*}}=0
$$

for all $x \in \mathbf{R}^{n} \backslash E$ (cf. Meyers [11], Bagby and Ziemer [3], and Michael and Ziemer [12]).

(ii) For each $\varepsilon>0$ there exists a closed set $F$ such that

$$
R_{k-m, p}\left(\mathbf{R}^{n} \backslash F\right)<\varepsilon \quad\left(\left|\mathbf{R}^{n} \backslash F\right|<\varepsilon\right)
$$

and

$$
\lim _{r \rightarrow 0} r^{-m}\left(f_{B(x, r)}\left|u(y)-P_{x}^{(m)}(y)\right|^{p^{*}} d y\right)^{1 / p^{*}}=0
$$

uniformly on $F$ (cf. Ziemer [15]).

Note that Theorem 4.3 is true also for $p^{*}=1$. We shall apply this to prove the Michael-Ziemer theorem.

Theorem 4.4 (Michael and Ziemer [12]). Let $\Omega$ be an open subset of $\mathbf{R}^{n}$ and let $k$ and $m$ be integers with $0 \leq m<k,(k-m) p<n$, where $1 \leq p<\infty$. Then, for $u \in W^{k, p}(\Omega)$ and each $\varepsilon>0$ there exists $v \in C^{m}(\Omega)$ such that

$$
R_{k-m, p}(\{x \in \Omega: u(x) \neq v(x)\})<\varepsilon \quad \text { and } \quad\|u-v\|_{m, p ; \Omega}<\varepsilon .
$$

We need the following lemmas for the proof of Theorem 4.4

Lemma 4.5. Let $k$ be a nonnegative integer. Then there exists $\varphi \in C_{0}^{\infty}\left(\mathbf{R}^{n}\right)$ with $\operatorname{supp} \varphi \subseteq B(0 ; 1)$ such that for every polynomial $P$ on $\mathbf{R}^{n}$ of degree at most $k$ and every $\varepsilon>0$,

$$
\varphi_{\varepsilon} * P=P
$$

where $\varphi_{\varepsilon}(x)=\varepsilon^{-n} \varphi(x / \varepsilon)$.

This lemma is due to Calderón and Zygmund [5]. See Liu and Tai 9 for another proof.

Lemma 4.6. Let $u \in L_{\text {loc }}^{1}\left(\mathbf{R}^{n}\right)$ and let $F$ be a closed subset of $\mathbf{R}^{n}$. If there exist a family of polynomials $\left\{P_{x}\right\}_{x \in F}$ of degree at most $k$ and a positive constant $M$ so that

$$
\left|D^{\alpha} P_{x}(x)\right| \leq M, \quad \sup _{r>0} r^{-k} f_{B(x, r)}\left|u(y)-P_{x}(y)\right| d y \leq M
$$

for $x \in F$, and

$$
\lim _{r \rightarrow 0} r^{-k} f_{B(x, r)}\left|u(y)-P_{x}(y)\right| d y=0
$$

uniformly on each compact subset of $F$, then $\left.u\right|_{F} \in t^{k}(F)$ with $\left\|\left.u\right|_{F}\right\|_{t^{k}(F)} \leq C M$, where $C$ is a positive constant depending only on $n$ and $k$. 
Proof. Choose a mollifier $\varphi$ as in Lemma4.5. For two distinct points $x$ and $y$ in $F$, let $\varepsilon=|x-y|$ and apply Lemma 4.5. Then

$$
\begin{aligned}
\left|D^{\alpha} P_{y}(y)-D^{\alpha} P_{x}(y)\right| & =\left|\left[\varphi_{\varepsilon} *\left(D^{\alpha} P_{y}-D^{\alpha} P_{x}\right)\right](y)\right| \\
& =\varepsilon^{-n-\alpha}\left|\int_{B(y, \varepsilon)}\left(D^{\alpha} \varphi\right)\left(\frac{y-z}{\varepsilon}\right)\left[P_{y}(z)-P_{x}(z)\right] d z\right| \\
\leq & \varepsilon^{k-|\alpha|} \omega_{n}\left\|D^{\alpha} \varphi\right\|_{\infty}\left[\varepsilon^{-k} f_{B(y, \varepsilon)}\left|u(z)-P_{x}(z)\right| d z\right. \\
& \left.+2^{n+k}(2 \varepsilon)^{-k} f_{B(x, 2 \varepsilon)}\left|u(z)-P_{y}(z)\right| d z\right] .
\end{aligned}
$$

Thus, $\lim _{y \rightarrow x}\left|D^{\alpha} P_{y}(y)-D^{\alpha} P_{x}(y)\right| /|y-x|^{k-|\alpha|}=0$ uniformly on each compact subset of $F$ and

$$
\left|D^{\alpha} P_{y}(y)-D^{\alpha} P_{x}(y)\right| \leq|x-y|^{k-|\alpha|}\left[\omega_{n}\|\varphi\|_{k, \infty}\left(2^{n+k}+1\right)\right] M
$$

whenever $x, y \in F,|\alpha| \leq k$. Therefore, $\left.u\right|_{F} \in t^{k}(F)$ with $\left\|\left.u\right|_{F}\right\|_{t^{k}(F)} \leq C(n, k) M$. The proof is complete.

Proof of Theorem 4.4. Initially, it will be assumed that $\Omega=\mathbf{R}^{n}$. For $\lambda>0$, let $W_{\lambda}=\left\{x \in \mathbf{R}^{n}: \sum_{|\alpha| \leq m} M\left(D^{\alpha} u\right)(x) \leq \lambda\right\}$. Then, it follows from (2.1), (2.2) and Theorem 1.2 that

$$
\begin{aligned}
& R_{k-m, p}\left(\mathbf{R}^{n} \backslash W_{\lambda}\right) \leq C(n, k, p)\|u\|_{k, p}^{p} / \lambda^{p}, \\
& \left|\mathbf{R}^{n} \backslash W_{\lambda}\right| \leq C(n, k, p)\|u\|_{k, p}^{p} / \lambda^{p}
\end{aligned}
$$

and

$$
\lim _{\lambda \rightarrow \infty} \lambda^{p}\left|\mathbf{R}^{n} \backslash W_{\lambda}\right|=0 .
$$

According to Theorem 4.1 and Theorem 4.3, we can find a closed set $F_{\lambda} \subseteq W_{\lambda}$ such that

$$
\begin{aligned}
& R_{k-m, p}\left(\mathbf{R}^{n} \backslash F_{\lambda}\right) \leq 2 C(n, k, p)\|u\|_{k, p}^{p} / \lambda^{p}, \\
& \left|\mathbf{R}^{n} \backslash F_{\lambda}\right| \leq 2 C(n, k, p)\|u\|_{k, p}^{p} / \lambda, \\
& \text { and } \lim _{\lambda \rightarrow \infty} \lambda^{p}\left|\mathbf{R}^{n} \backslash F_{\lambda}\right|=0 \\
& \lim _{r \rightarrow 0} r^{-m} f_{B(x, r)}\left|D^{\alpha} u(y)-D^{\alpha} u(x)\right| d y=0
\end{aligned}
$$

for $x \in F,|\alpha| \leq m$;

$$
\lim _{r \rightarrow 0} r^{-m} f_{B(x, r)}\left|u(y)-P_{x}(y)\right| d y=0
$$

uniformly on $F_{\lambda}$; and

$$
\begin{aligned}
\sup _{r>0} r^{-m} f_{B(x, r)}\left|u(y)-P_{x}(y)\right| d y & \leq 2 \sum_{|\alpha|=m} M\left(D^{\alpha} u\right)(x) \\
& \leq 2 \lambda \quad \text { for } x \in F_{\alpha},
\end{aligned}
$$


where $P_{x}(y)=\sum_{|\alpha| \leq m} \frac{D^{\alpha} u(x)}{\alpha !}(y-x)^{\alpha}$. Thus, by virtue of Lemma 4.6] it follows from (4.2), (4.3) and (4.4) that $\left.u\right|_{F_{\lambda}} \in t^{m}\left(F_{\lambda}\right)$ with $\left\|\left.u\right|_{F_{\lambda}}\right\|_{t^{m}\left(F_{\lambda}\right)} \leq C(n, k) \lambda$. By the Whitney extension theorem, there exists $u_{\lambda} \in C^{m}\left(\mathbf{R}^{n}\right)$ such that

$$
\left.u_{\lambda}\right|_{F_{\lambda}}=\left.u\right|_{F_{\lambda}} \text { in } t^{m}\left(F_{\lambda}\right) \quad \text { and } \quad\left\|u_{\lambda}\right\|_{m, \infty} \leq(n, k) \lambda .
$$

Hence, $\left\{x \in \mathbf{R}^{n}: u_{\lambda}(x) \neq u(x)\right\} \subseteq \mathbf{R}^{n} \backslash F_{\lambda}$. From (4.1), we then have

$$
\begin{aligned}
R_{k-m, p}\left(\left\{x \in \mathbf{R}^{n}: u_{\lambda}(x) \neq u(x)\right\}\right) & \leq R_{k-m, p}\left(\mathbf{R}^{n} \backslash F_{\lambda}\right) \\
& \leq 2 C(n, k, p)\|u\|_{k, p}^{p} / \lambda^{p} \rightarrow 0,
\end{aligned}
$$

as $\lambda \rightarrow \infty$. Besides, for $|\alpha| \leq m$,

$$
\begin{aligned}
\left\|D^{\alpha} u_{\lambda}-D^{\alpha} u\right\|_{p} & =\left\|D^{\alpha} u_{\lambda}-D^{\alpha} u\right\|_{p ; \mathbf{R}^{n} \backslash F_{\lambda}} \quad(\text { by }(4.5)) \\
& \leq\left\|D^{\alpha} u\right\|_{p ; \mathbf{R}^{n} \backslash F_{\lambda}}+\left\|D^{\alpha} u_{\lambda}\right\|_{p ; \mathbf{R}^{n} \backslash F_{\lambda}} \\
& \leq\left\|D^{\alpha} u\right\|_{p ; \mathbf{R}^{n} \backslash F_{\lambda}}+C(n, k, p) \lambda\left|\mathbf{R}^{n} \backslash F_{\lambda}\right|^{1 / p} \rightarrow 0
\end{aligned}
$$

as $\lambda \rightarrow \infty$ (by (4.1)). Combining (4.6) and (4.7), we have completed the proof for the case $\Omega=\mathbf{R}^{n}$.

The general case follows by using the technique of a partition of unity as in Ziemer [16, Theorem 3.11.6].

Remark 4.7. Let $B_{k, p}$ be the Bessel capacity on $\mathbf{R}^{n}$, where $k>0,1<p<\infty$, $k p \leq n$ (see Ziemer [16] for the definition of Bessel capacity). Then, it is a wellknown result that

$$
B_{k, p}\left(\left\{x \in \mathbf{R}^{n}: M u(x)>\lambda\right\}\right) \leq C(n, k, p)\|u\|_{k, p}^{p} / \lambda^{p}
$$

for $\lambda>0$ and $u \in W^{k, p}\left(\mathbf{R}^{n}\right)$ (see Ziemer [16, Remark 3.10.3] or Adams [1]). If we replace Theorem 1.2 by (4.8), following the proof of Theorem 4.1, Theorem 4.3 and Theorem 4.4, we obtain the following result:

Let $\Omega$ be an open subset of $\mathbf{R}^{n}$ and let $k, m$ be integers such that $0 \leq m<k$, $(k-m) p \leq n$, where $1<p<\infty$. Then, for $u \in W^{k, p}(\Omega)$ and each $\varepsilon>0$ there exists $v \in C^{m}(\Omega)$ such that

$$
B_{k-m, p}(\{x \in \Omega: u(x) \neq v(x)\})<\varepsilon \text { and }\|u-v\|_{m, p ; \Omega}<\varepsilon .
$$

This result is equivalent to Theorem 4.4 when $0 \leq m<k,(k-m) p<n$ and $1<p<\infty$ (see Ziemer [16, Remark 3.11.7]). But the result is new in the case $(k-m) p=n$.

\section{REFERENCES}

1. D. R. Adams, Maximal operator and capacity, Proc. Amer. Math. Soc., 34 (1972), 152-156. MR 50:2807

2. D. R. Adams, Quasi-additivity and sets of finite $L^{\varphi}$-capacity, Pacific J. Math., 79 (1978), 283-291. MR 81j:31006

3. T. Bagby and W. P. Ziemer, Pointwise differentiability and absolute continuity, Trans. Amer. Math. Soc. 191 (1974), 129-148. MR 49:9129

4. A. P. Calderón, Lebesgue spaces of differentiable functions and distributions, Proc. Symp. Pure Math., IV (1961), 33-49. MR 26:603

5. A. P. Calderón and A. Zygmund, Local properties of solutions of elliptic partial differential equations, Studia Math., 20 (1961), 171-225. MR 25:310

6. H. Federer, Geometric Measure Theory, Springer-Verlag, New York, Heidelberg, 1969. MR 41:1976

7. H. Federer and W. P. Ziemer, The Lebesgue set of a function whose distribution derivatives are pth power summable, Ind. Univ. Math. J., 22 (1972), 139-158. MR 55:8321 
8. Fon-Che Liu, A Lusin type property of Sobolev functions, Ind. Univ. Math. J., 26 (1977), 645-651.

9. Fon-Che Liu and Wei-Shyan Tai, Maximal Steepness and Lusin type properties, Ric. Mat., 43 (1994), 365-384. |MR 96c:26013

10. B. Malgrange, Ideals of Differentiable Functions, Oxford University Press, 1966. MR 35:3446

11. N. Meyers, Taylor expansion of Bessel potentials, Indiana Univ. Math. J., 23 (1974), 10431049. MR 50:980

12. J. Michael and W. P. Ziemer, A Lusin type approximation of Sobolev functions by smooth functions, Contemporary Mathematics, AMS, 42 (1985), 135-167. MR 87e:46051

13. E. M. Stein, Singular Integrals and Differentiability Properties of Functions, Princeton University Press, 1970. MR 44:7280

14. H. Whitney, Analytic extensions of differentiable functions defined in closed sets, Trans. Amer. Math. Soc., 36 (1934), 63-89. CMP 95:18

15. W. P. Ziemer, Uniform differentiability of Sobolev functions, Ind. Univ. Math. J., 37 (1988), 789-699. MR 90f: 46054

16. W. P. Ziemer, Weakly Differentiable Functions, Springer-Verlag, New York, 1989. MR 91e:46046

Department of Mathematics, National Chung Cheng University, Mingshiung, Chai Yi 61117, TAIWAN, R.O.C. 